International Journal of Surgery and Research (1JSR)

ISSN: 2379-156X

\title{
Guided Regeneration of Nerve Repair by Means of Local Administration of Insulin-Like Growth Factor I In Streptozotocin Induced Diabetes In Rats \\ Research Article
}

\author{
Mohammadi $\mathrm{R}^{1 *}$, Rostami $\mathrm{H}^{1}$, Payman $\mathrm{T}^{1}$, Salam $\mathrm{E}^{1}$, Salehi $\mathrm{H}^{1}$, Aliyali $\mathrm{P}^{1}$, Soleimani $\mathrm{H}^{1}$, Amini $\mathrm{K}^{2}$ \\ ${ }^{1}$ Department of Clinical Sciences, Faculty of Veterinary Medicine, Urmia University, Iran. \\ ${ }^{2}$ Department of Veterinary Pathology, Western College of Veterinary Medicine, University of Saskatchewan, Canada.
}

\begin{abstract}
The aim was to evaluate the effect of locally administered insulin-like growth factor (IGF I) on functional recovery of peripheral nerve in diabetic rats. Sixty male healthy white Wistar rats were divided into four experimental groups $(n=15)$, randomly: In transected group (TC), left sciatic nerve was transected and stumps were fixed in the adjacent muscle. In treatment group defect was bridged using a silicone conduit (SIL/IGF) and filled with $10 \mu \mathrm{L} \mathrm{IGF} \mathrm{I} \mathrm{(100} \mathrm{ng/kg).} \mathrm{In} \mathrm{silicone} \mathrm{graft}$ group (SIL), the graft was filled with phosphate-buffered saline alone. In sham-operated group (SHAM), sciatic nerve was exposed and manipulated. Each group was subdivided into three subgroups of five animals each and regenerated nerve fibers were studied 4, 8 and 12 weeks after surgery. Behavioral testing, sciatic nerve functional study, gastrocnemius muscle mass and morphometric indices confirmed faster recovery of regenerated axons in SIL/IGF than in SIL group $(p<0.05)$. In immunohistochemistry, location of reactions to S-100 in SIL/IGF was clearly more positive than that in SIL group. When loaded in a silicone graft, IGF I accelerated and improved functional recovery and morphometric indices of sciatic nerve in diabetic rats.
\end{abstract}

Keywords: Peripheral Nerve Repair; Sciatic; Insulin-Like Growth Factor I; Local.

\section{*Corresponding Author:}

Rahim Mohammadi D.V.M., D.V.Sc.,

Department of Clinical Sciences, Faculty of Veterinary Medicine, Urmia University, Nazloo Road, Urmia, 57153 1177, Iran.

Tel: +984412770508

Fax: +984412771926

E-mail: r.mohammadi@urmia.ac.ir

Received: January 31, 2015

Accepted: April 07, 2015

Published: April 10, 2015

Citation: Mohammadi R, et al., (2015) Guided Regeneration of Nerve Repair by Means of Local Administration of Insulin-Like Growth Factor I In Streptozotocin Induced Diabetes In Rats. Int J Surg Res 2(3) 13-20. doi: http://dx.doi.org/10.19070/2379-156X-150004

Copyright: Mohammadi $\mathbf{R}^{\circ}$ 2015. This is an open-access article distributed under the terms of the Creative Commons Attribution License, which permits unrestricted use, distribution and reproduction in any medium, provided the original author and source are credited.

\section{Introduction}

Organ reinnervation and functional recovery following peripheral nerve injury still is a major challenge and return of functional recovery to the pre injury level rarely occurs [1]. Recent therapeutic advances in the control of diabetes mellitus and diabetic neuropathy have renewed the interest in the rate and quality of nerve regeneration in this chronic disease [2]. Although measurable improvements may follow better control of blood sugar and administration of aldose reductase inhibitors, complete recovery is dependent on the regeneration of damaged axons and the rees- tablishment of fully functional connection with their targets [3].

Peripheral nerve contains both dendrites and axons which conduct information to (afferent) or from (efferent) the CNS, respectively. Although the smallest axons are unmyelinated, most axons in a peripheral nerve are myelinated with the multiple concentric layers of lipid-rich with biochemically modifying plasma membrane produced by Schwann cells. Schwann cells and their endoneurial extracellular matrix play pivotal roles in the selective promotion of motor and sensory axon regeneration [4]. When an axon is crushed or severed, changes occur on both sides of the lesion [5]. Where a gap is present between the severed ends of the nerve, proliferating Schwann cells emerge from the stumps (mainly the distal stump) and form series of nucleated cellular cords (the bands of Bungner) which bridge the interval [6].

Widely accepted method by most surgeons is bridging the defect with an autologous donor nerve. To achieve maximum functional recovery various techniques are being used. Different graft equivalents have also been applied to bridge the nerve stump and regulated through the interaction of a variety of protein and cell signals [7].

The conduits act to guide axons sprouting from the regenerating nerve end, provide a microenvironment for diffusion of neurotrophic and neurotropic factors secreted by the injured nerve stump, as well as help protect infiltration of fibrous tissue [8].

It has been reported that using silicone tubes in bridging of nerve defects could be promising because it is inert and does not induce extensive scarring or degeneration after implantation [9]. The ad- 
vantages like no donor morbidity, availability, affordability and no foreign reactions make silicone rubber chamber an attractive alternative compared to other standard grafts [10]. It has been demonstrated that silicone rubber tubes are well tolerated in humans even after 3 years of implantation [11]. Silicone chambers are used as standard experimental model to study the nerve regeneration process [12].

Neurotrophic factors are a family of growth factors that support and influence the growth and regenerative capacity of neurons. These substances are produced by tissues during development and direct the formation of the brain and spinal cord and their connections to target organs such as muscle [13-15]. In adults IGF-I immunoreactivity is detectable in the ventral horn, sympathetic and dorsal root ganglia in the adult rat, and axons and Schwann cells of the sciatic nerve $[16,17]$. During development, both motor and sensory neurons also respond to IGF-I and -II with increased neurite outgrowth [17,18-21]. Local injection of either IGF-I or -II increase levels of growth associated protein (GAP)-43 and sprouting of intramuscular nerve fibers [22]. IGF-1, a single-stranded polypeptide with 70 amino acids composition, belongs to the family of Insulin-like growth factor and shows endocrine, autocrine and paracrine activities. Its molecular weight is about $7649 \mathrm{~Kb}$. Since the first report from Salmon and Daughaday, this factor that is closely related to growth hormone, has attracted researches in many fields, and the appearance of bio products of recombination of IGF-1 further has opened the research visions in the fields of disease diagnosis and therapy [23-25]. IGF-I signalings through the type I IGF receptor, exerts potent effects on neuronal growth, survival, and processes outgrowth $[26,27]$. Schwann cell is an essential cellular component of peripheral nerve and a source of IGF after nerve injury. IGFs are also important factors in Schwann cell biology. IGF-I is a potent mitogen for Schwann cells in culture [28-30]. IGF-I promotes not only the expression of myelin proteins such as Protein zero but also segmental myelination of dorsal root ganglia axons in culture, including the formation of nodes of Ranvier [31]. Promising results regarding beneficial effect of IGF I on peripheral nerve regeneration is not supported by functional tests, to the best knowledge of the authors, which paly a crucial role in assessment of functional nerve recovery.

However, to the best knowledge of the authors literature is poor concerning effects of combination of silicone conduit and IGF I on peripheral nerve regeneration in vivo in diabetic rats. The objective of the present study was to evaluate effectiveness of IGF I on peripheral nerve regeneration using a diabetic rat sciatic nerve transection model. Therefore, a silicone conduit was prepared and filled with IGF I. Assessment of the nerve regeneration was based on behavioral, functional (Walking Track Analysis), muscle mass measurement, histomorphometric and immuohistochemical (Schwann cell detection by S100 expression) criteria 4, 8 and 12 weeks after surgery.

\section{Materials and Methods}

\section{Study Design and Animals}

Sixty male Wistar rats weighing approximately $300 \mathrm{~g}$ were divided into four experimental groups $(n=15)$, randomly: sham-operation group as normal control (SHAM), transected control (TC), silicone conduit (SIL) and IGF treated group (SIL/IGF). Each group was further subdivided into three subgroups of five animals each and surveyed 4, 8 and 12 weeks after surgery. Two weeks before and during the experiments, the animals were housed in individual plastic cages with an ambient temperature of $(23 \pm 3)^{\circ}$ $\mathrm{C}$, stable air humidity and a natural day/night cycle. The rats had free access to standard rodent laboratory food and tap water. All measurements were made by two blinded observers unaware of the analyzed groups.

\section{Surgical Procedure}

Animals were anesthetized by intraperitoneal administration of ketamine-xylazine (ketamine 5\%, 90mg/kg and xylazine $2 \%, 5 \mathrm{mg} / \mathrm{kg}$ ). The procedure was carried out based on the guidelines of the Ethics Committee of the International Association for the Study of Pain [32]. The University Research Council approved all experiments.

Following surgical preparation in the sham-operation group, the left sciatic nerve was exposed through a gluteal muscle incision and after careful homeostasis the muscle was sutured with resorbable 4/0 sutures, and the skin with $3 / 0$ nylon. In TC group, the left sciatic nerve was transected proximal to the tibio-peroneal bifurcation where a $7 \mathrm{~mm}$ segment was excised, leaving a $10 \mathrm{~mm}$ gap due to retraction of nerve ends. Proximal and distal stumps were fixed in the adjacent muscle with 10/0 nylon epineurial suture. No graft was interposed between the stumps. In control group (SIL), the left sciatic nerve was exposed the same way, transected proximal to the tibio-peroneal bifurcation where a $7 \mathrm{~mm}$ segment was excised, leaving a gap about $10 \mathrm{~mm}$ due to retraction of the nerve ends. The proximal and distal stumps were each inserted 2 $\mathrm{mm}$ into the conduit and two 10/0 nylon sutures were placed at each end of the cuff to fix the tube in place and leave a $10-\mathrm{mm}$ gap between the stumps. The silicone tube was filled with $10 \mu \mathrm{L}$ phosphate buffered solution. Sterile Vaseline was used to seal the ends of the tubes to avoid leakage. In IGF treated group (SIL/ IGF) the silicone graft was filled with $10 \mu \mathrm{l}$ IGF I (100ng/kg). All surgical procedures were carried out by the same surgeon, using a sterile microsurgical technique. After surgery had carried out animals were housed in groups of five per cage under the same conditions mentioned above. The animals were anesthetized and euthanized with transcardiac perfusion of a fixative containing $2 \%$ paraformaldehyde and 1\%glutaraldehyde buffer ( $\mathrm{pH} 7.4$ ) 4, 8 and 12 weeks after surgery.

\section{Behavioral Testing}

Functional recovery of the nerve was assessed using the Basso, Beattie, and Bresnahan (BBB) locomotor rating scale for rat hind limb motor function [33]. Although BBB is widely used to assess functional recovery in spinal cord injured animals, however, it has been demonstrated that it could be most useful in assessment of never repair processes in peripheral nerve injuries [1]. Scores of 0 and 21 were given when there were no spontaneous movement and normal movement, respectively. A score of 14 shows full weight support and complete limbs coordination. BBB recordings were performed by a trained observer who was blinded to the experimental design. The testing was performed in a serene environment. The animals were observed and assessed within a course of a 4-minute exposure to an open area of a mental circular enclosure. BBB scores were recorded once before surgery in order to establish a baseline control and again weekly thereafter to assess functional recovery during 12 weeks. 


\section{Functional Assessment of Reinnervation}

Sciatic functional index (SFI): Walking track analysis was performed 4, 8 and 12 weeks after surgery based on the method of others [19]. The lengths of the third toe to its heel (PL), the first to the fifth toe (TS), and the second toe to the fourth toe (IT) were measured on the experimental side $(\mathrm{E})$ and the contralateral normal side $(\mathrm{N})$ in each rat. The sciatic function index (SFI) of each animal was calculated by the following formula:

\section{SFI $=-38.3 \times($ EPL-NPL $) /$ NPL $+109.5 \times($ ETS-NTS $) / N T S$ $+13.3 \times($ EIT-NIT $) /$ NIT -8.8}

In general, SFI oscillates around 0 for normal nerve function, whereas around -100 SFI represents total dysfunction. SFI was assessed in the NC group and the normal level was considered as 0 . SFI was a negative value and a higher SFI meant the better function of the sciatic nerve.

Static sciatic index (SSI): SSI is a time-saving digitized static footprint analysis described by others [34]. A good correlation between the traditional SFI and the newly developed static sciatic index (SSI) and static toe spread factor (TSF), respectively, has been reported by others [34]. The SSI is a time-saving and easy technique for accurate functional assessment of peripheral nerve regeneration in rats and is calculated using the static factors, not considering the print length factor (PL), according to the equation:

$\mathrm{SSI}=[(108.44 \times \mathrm{TSF})+(31.85 \times \mathrm{ITSF})]-5.49$

Where:

$\mathrm{TSF}=(\mathrm{ETS}-\mathrm{NTS}) / \mathrm{NTS}$

$\mathrm{ITSF}=(\mathrm{EIT}-\mathrm{NIT}) / \mathrm{NIT}$

Like SFI, an index score of 0 was considered normal and an index of -100 indicated total impairment. When no footprints were measurable, the index score of -100 was given.

\section{Muscle Mass}

Recovery assessment was also indexed using the weight ratio of the gastrocnemius muscles 12 weeks after surgery. Immediately after sacrificing of animals, gastrocnemius muscles were dissected and harvested carefully from intact and injured sides and weighed while still wet, using an electronic balance.

\section{Histological Preparation and Morphometric Studies}

Nerve mid-substance in SIL group, nerve mid-substance in IGF I treated group, midpoint of normal sciatic nerve (Sham) and regenerated mid substance of TC group were harvested and fixed with glutaraldehyde $2.5 \%$. They were post fixed in OsO4 $(2 \%, 2$ h), dehydrated through an ethanol series and embedded in Epon. The nerves were cut in $5 \mu \mathrm{m}$ in the middle, stained with toluidine blue and examined under light microscopy. Morphometric analysis was carried out using an image analyzing software (Image-Pro Express, version 6.0.0.319, Media Cybernetics, Silver Springs, MD, USA). Equal opportunity, systematic random sampling and two-dimensional dissector rules were followed in order to cope with sampling-related, fiber-location-related and fiber-size related biases [35].

\section{Immunohistochemical Analysis}

In this study, anti-S-100 (1:200, DAKO, USA) was used as marker for myelin sheath. Specimens were post fixed with 4\% paraformaldehyde for $2 \mathrm{~h}$ and embedded in paraffin. Prior to immunohistochemistry nerve sections were dewaxed and rehydrated in PBS $(\mathrm{pH}$ 7.4). Then the nerve sections were incubated with $0.6 \%$ hydrogen peroxide for 30 minutes. To block non-specific immunoreactions the sections were incubated with normal swine serum (1:50, DAKO, USA). Sections were then incubated in S-100 protein antibody solution for $1 \mathrm{~h}$ at room temperature. They were washed three times with PBS and incubated in biotynilated anti-mouse rabbit IgG solution for $1 \mathrm{~h}$. Horseradish peroxidaselabelled secondary antibody was applied for $1 \mathrm{~h}$. After that all sections were incubated with 3,3'- diaminobenzidine tetrahydrochloride chromogene substrate solution (DAB, DAKO, USA) for 10 min. The results of immunohistochemistry were examined under a light microscope.

\section{Statistical Analysis}

The results were expressed as means \pm SD. Statistical analyses were performed using PASW 18.0 (SPSS Inc., Chicago, IL, USA). Model assumptions were evaluated by examining the residual plot. Results were analyzed using a factorial ANOVA with two between-subjects factors. Bonferroni test for pairwise comparisons was used to examine the effect of time and treatments. The differences were set at $\mathrm{P}<0.05$.

\section{Results}

\section{Behavioral Testing}

$\boldsymbol{B B B}$ recovery: In order to assess hind limb recovery the open field locomotor was used. Figure. 1 shows BBB scores compared to the baseline. All experimental groups, except for sham, showed the greatest degree of functional deficit one week after surgery. The IGF I treated group showed significant improvement in locomotion of the operated limb compared to the SIL group during the study period $(\mathrm{P}<0.05)$.

\section{Recovery of Sciatic Nerve Function}

SFI Outcome: Figure. 2 shows sciatic function index (SFI) values in all four experimental groups. Prior to surgery, SFI values in all groups were near zero. After the nerve transection, the mean SFI decreased to -100 due to the complete loss of sciatic nerve function in all animals. At the end of the study period, animals of IGF I group achieved a mean value for SFI of $-45.8 \pm-2.36$ whereas in group SIL a mean value of $-63.3 \pm-3.14$ was found. The statistical analyses revealed that the recovery of nerve function was significantly $(\mathrm{P}<0.05)$ different between SIL/IGF and SIL groups and application of the IGF I in silicone conduit significantly accelerated functional recovery in the course of time.

SSI outcome: Changes in SSI were similar to those observed in SFI, indicating significant deficit following the sciatic nerve transection (Figure 3). Changes in SSI were significant at weeks 4, 8 and 12 of recovery $(\mathrm{P}<0.05)$. The contrasts indicate SSI values in group SIL/IGF at week 12 to differ significantly from those obtained from SIL, a trend also noticed for SFI $(\mathrm{P}<0.05)$. 
The mean ratios of gastrocnemius muscle weight were measured at the end of the study period. There was a statistically significant difference between the muscle weight ratios of the SIL/IGF and SIL groups $(\mathrm{P}<0.05)$. The results showed that in the IGF I treated group, the muscle weight ratio was larger than in the SIL group, and weight loss in the gastrocnemius muscle was ameliorated by IGF I local administration (Figure 4).

\section{Histological and Morphometric findings}

The IGF I treated group presented significantly greater nerve fiber, axon diameter, and myelin sheath thickness during study period, compared to SIL animals $(\mathrm{P}<0.05)$. Sham-operation group presented significantly greater nerve fiber and axon diameter, and myelin sheath thickness compared to SIL/IGF and SIL groups animals (Figure 5-8). In case of myelin thickness there was no significant difference between SIL/IGF and SIL groups, morphometrically $(\mathrm{P}>0.05)$.
Immunoreactivity to S-100 protein was extensively observed in the cross sections of regenerated nerve segments. The expression of S-100 protein signal was located mainly in the myelin sheath. The axon also showed a weak expression indicating that Schwann cell-like phenotype existed around the myelinated axons. In both SIL/IGF and SIL groups, the expression of S-100 and the findings resembled those of the histological evaluations.

\section{Discussion}

A few reports have been carried out about Wallerian degeneration after nerve injury in experimental diabetes postulating that Wallerian degeneration is delayed in diabetes $[36,37]$. Despite much effort to introduce ideal therapeutic drugs for diabetic neuropathy, aldose reductase inhibitors, have been shown to be the most established compounds among potent drugs. However, although experimental data on aldose reductase inhibitors have been very promising, their clinical efficacy seems limited even for mild degrees of diabetic neuropathy [27].

Figure 1. BBB score for all experimental groups. Local administration of IGF I with silicone grafting gave better scores than in SIL group. Standard error at each data point is shown with bars.

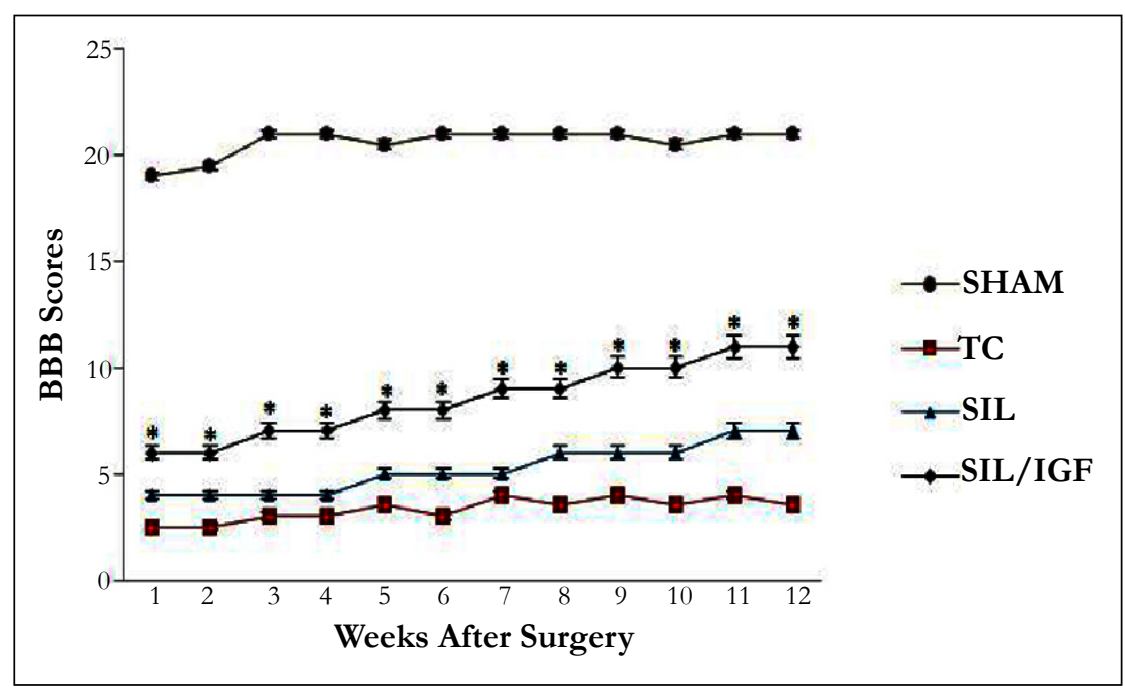

Figure 2. Box-and-whisker plots of sciatic nerve function index values in each experimental group during the study period. Local administration of IGF I with silicone grafting gave better results in functional recovery of the sciatic nerve than in SIL group.

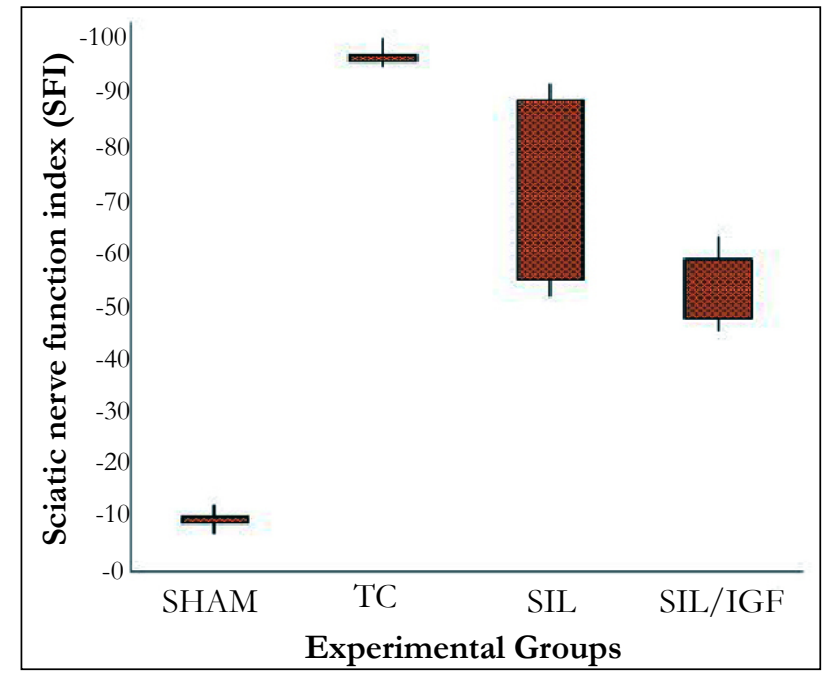


Figure 3. Bar graph indicating static sciatic index (SSI) values in each experimental group during the study period. Local administration of IGF I with silicone grafting gave better results in functional recovery of the sciatic nerve than in SIL group. Data are presented as mean \pm SD. $* P<0.05$ vs SIL group.

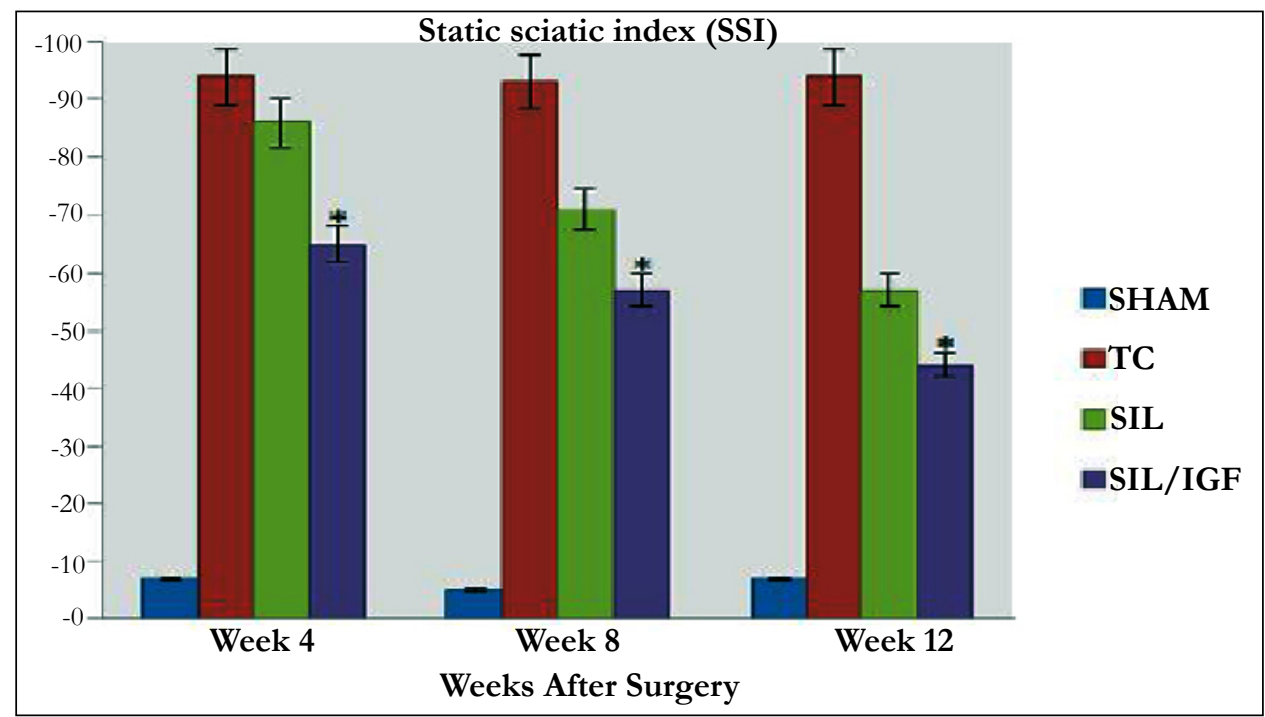

Figure. 4 Gastrocnemius muscle weight measurement. The gastrocnemius muscles of both sides (operated left and unoperated right) were excised and weighed in the experimental groups at 12 weeks after surgery. Data are presented as mean \pm

SD. $* \mathbf{P}<0.05$ vs SIL group.

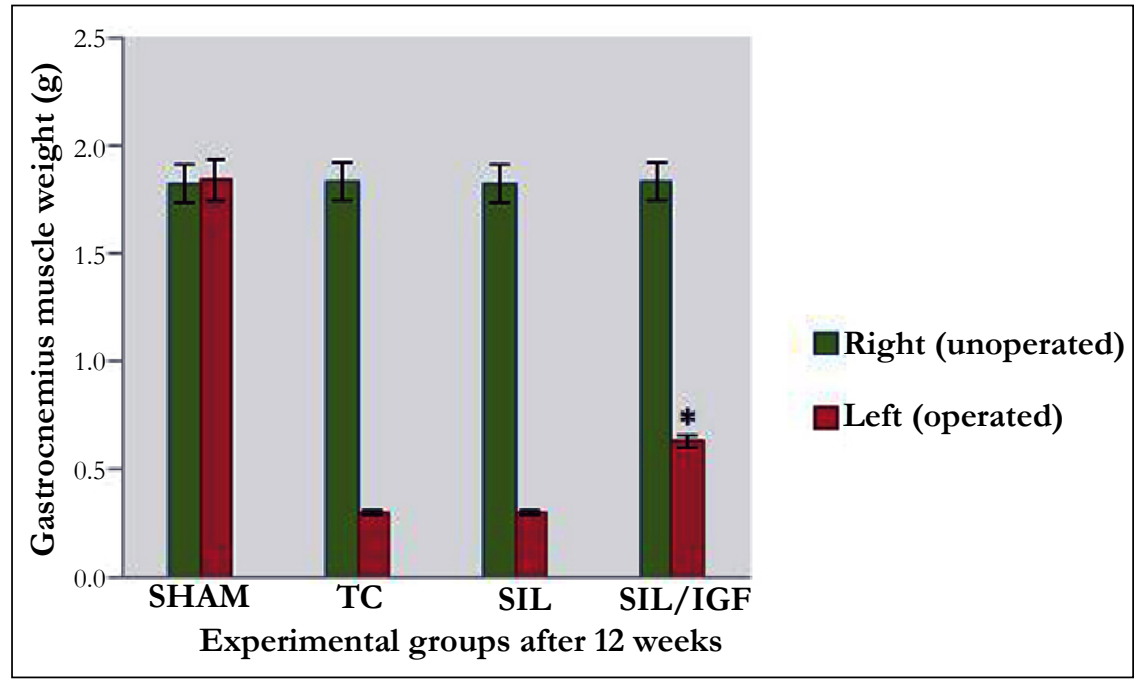

Figure 5. The graph shows the quantitative results of fiber counting. The mean number of nerve fibers in SHAM group was nearly $8204 \pm 208$ (mean \pm SD). Both groups of SIL and SIL/IGF showed the lower number of fibers than the shamoperated group even at the end of the study period.

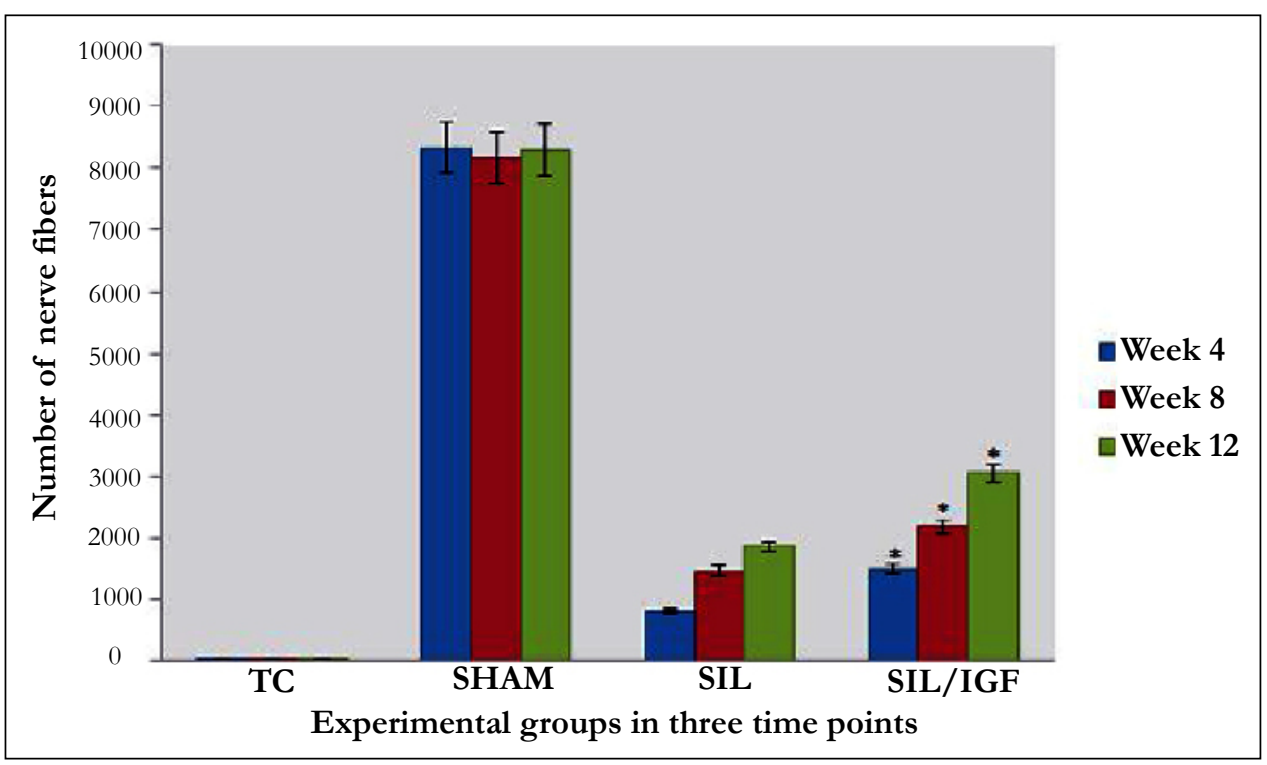


Figure 6. The graph shows the quantitative results of mean diameter of nerves fibers. The mean diameter of nerve fibers in SHAM group was nearly $12.5 \pm 0.17$ (mean \pm SD). Both groups of SIL and SIL/IGF showed the lower mean diameter of nerve fibers than the sham-operated group even at the end of the study.

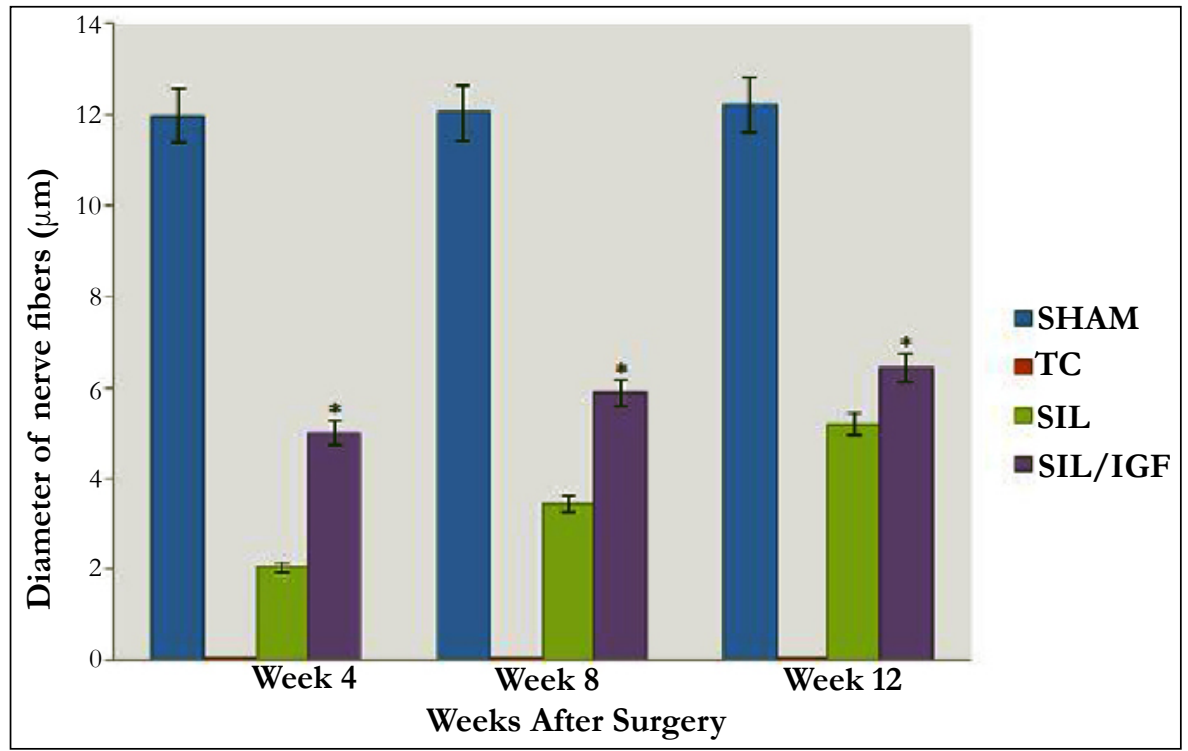

Figure 7. The graph shows the quantitative results of mean thickness of myelin sheath. The mean thickness of myelin sheath in SHAM group was nearly $2.5 \pm 0.04$ (mean $\pm \mathrm{SD}$ ). Both groups of SIL and SIL/IGF showed the lower mean diameter of axons than the sham-operated group even at the end of the study period.

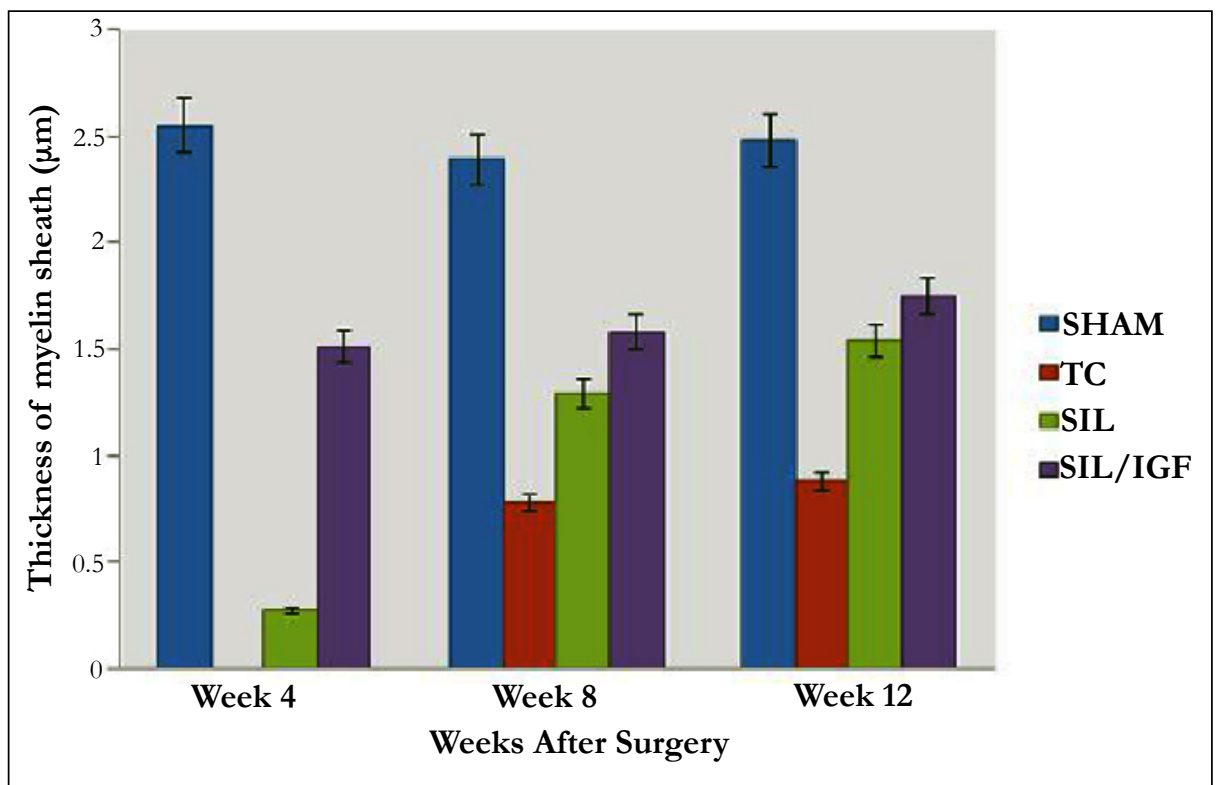

Figure 8. Immunohistochemical analysis of the regenerated nerves 12 weeks after surgery from (A) middle cable TC, (B) SIL, (C) SIL/IGF and (D) SHAM. There is clearly more positive staining of the myelin sheath-associated protein S- 100 (arrows) within the periphery of nerve, indicating well organized structural nerve reconstruction in IGF I treated nerve compared to that of the SIL. Scale bar: $10 \mu \mathrm{m}$.

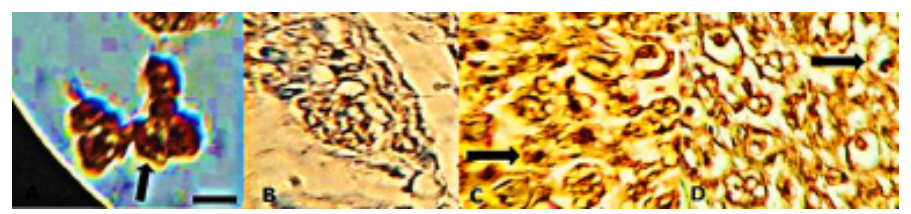

The results of the present study showed that application of IGF $\mathrm{I}$ in a silicone graft resulted in faster functional recovery of the sciatic nerve in diabetic rats during the study period. Left gastrocnemius muscle weight was significantly greater in the SIL/ IGF group than in the SIL group, indicating indirect evidence of successful end organ reinnervation in the IGF I treated animals. At week 12 quantitative morphometrical indices of regenerated nerve fibers showed significant differences between the SIL and
SIL/IGF groups, indicating a beneficial effect of local application of IGF I on the nerve regeneration.

Although both morphological and functional data have been used to assess neural regeneration after induced crush injuries, the correlation between these two types of assessment is usually poor [39-42]. Classical and newly developed methods of assessing nerve recovery, including histomorphometry, retrograde trans- 
port of horseradish peroxidase and retrograde fluorescent labeling [41] do not necessarily predict the reestablishment of motor and sensory functions [40, 44-46]. Although such techniques are useful in studying the nerve regeneration process, they generally fail in assessing functional recovery [40]. Therefore, research on peripheral nerve injury needs to combine both functional and morphological assessment. Castaneda et al, [46] suggested that arrival of sprouts from the proximal stump at the distal nerve stump does not necessarily imply recovery of nerve function. Information taken from BBB scale may be invaluable in evaluation of peripheral nerve process. Results of the present study showed that the IGF treated animals had been improved in locomotion of the operated limb compared to the SIL group during the study period. Walking track analysis has frequently been used to reliably determine functional recovery following nerve repair in rat models [19, 44].

A wide variety of materials have been used to produce nerve guides, including non-biodegradable and biodegradable materials. Because of its inert and elastic properties, the silicon tube was one of the first and most frequently used to bridge the transected nerves [47].

The accumulation of IGF-I after peripheral nerve damage could be the result of disrupted axonal transport or a local therapeutic response. The relevance of post injury up-regulation of IGF has been addressed in multiple studies. Others in an experimental study without being supported by functional tests concluded that local infusion of IGF-I at appropriate concentration promotes regeneration of a peripheral nerve [48]. Kanje et al. [49] reported that IGF-I significantly increased axonal regeneration by $49 \%$ in a rat sciatic nerve crush/ transection model. This effect was unique to IGF-I because nerve growth factor treatment had no effect. The in vivo mechanisms of IGF action are assumed to be similar to those activated in primary neuronal cultures. Studies applying pathway-specific inhibitors are not available, however, antibodies to IGF-I but not nerve growth factor or insulin have completely blocked the ability of IGF-I to promote axonal regrowth $[49,50]$. Further work by others has examined the effects of a preconditioning transection that is performed before nerve crush [51]. Under these conditions, regeneration was more robust than crush alone. This augmented regeneration was blocked by inhibitors of protein or RNA synthesis or retrograde axonal transport [51]. Perfusion of IGF-I is able to overcome the effects of cycloheximide (protein synthesis inhibitor), indicating that locally produced IGF-I is retrogradely transported to the cell body in which it exerts its positive effects [51].

Even though our preliminary study shows the neuroprotective action of local IGF I in peripheral nerve injuries, determining the molecular mechanisms leading to the neuroprotective action remains needs to be investigated. We have not given the histological and molecular evidence for neuroprotective action of IGF I. This may be considered as a limitation to our study. Therefore, the authors stress that the aim of the current investigation was to evaluate a single local dose and clinical treatment potential of IGF I on nerve regeneration including functional assessments of the nerve repair, a case not considered in previous studies. The results of the present study indicated that a single local administration of IGF I at the site of transected nerve could be of benefit after silicone tubulization in diabetic rats.

The present study demonstrated that a single local application of
IGF I could accelerated functional recovery after transection of sciatic nerve. Thus, dose-response studies should be conducted for IGF I to determine the combination of the graft and the compound that achieve maximal efficacy in nerve transection models in diabetic rats. Detailed mechanism of neuroprotective action of IGF I in diabetic rats where neutralizing antibodies against IGF I is performed remains to be investigated.

\section{Acknowledgement}

The authors would like to thank Mr. Matin and Mr. Valinezhad, Department of Clinical Sciences, and Mr. Jaafary, Urmia Pathobiology Center, for their technical expertise.

\section{References}

[1]. Dinh P, Hazel A, Palispis W, Suryadevara S, Gupta R (2009) Functional assessment after sciatic nerve injury in a rat model. Microsurg 29(8): 644 -649 .

[2]. Thomas PK, Eliasson SG (1984) Diabetic neuropathy. In: Dyck PJ, Thomas PK, Lambert EH, Bunge RP (eds) Peripheral Neuropathy, 2nd edn. Philadelphia, WB Saunders. 2: 1773-1810.

[3]. Longo FM, Powell HC, Lebeau J, Gerrero MR, Heckman H, et al. (1986) Delayed nerve regeneration in streptozotocin diabetic rats. Muscle Nerve 9(5): 385-393.

[4]. Elizabeth OJ, Aristides BZ. Panayotis NS (2005) Regeneration and repair of peripheral nerves. Injury 36(4): S24-S29.

[5]. Malizos KN, Dailiana ZH, Anastasiou EA, Anastasiou EA, Sarmas I, et al. (1997) Neuromas and gaps of sensory nerves of the hand: management using vein conduits. Am J Orthop (Belle Mead NJ) 26(7): 481-485.

[6]. Zhang Z, Soucacos PN, Beris AE, Bo J, Ioachim E, et al. (2000) Long-term evaluation of rat peripheral nerve repair with the method of end to- side neurorrhaphy. J Reconstr Microsurg 16(4): 303-311.

[7]. Evans GR (2001) Peripheral nerve injury: A review and approach to tissue engineered constructs. Anat Rec 263(4): 396 -404.

[8]. Quarles RH (2002) Myelin sheaths: glycoproteins involved in their formation, maintenance and degeneration.Cell Mol Life Sci 59(11): 1851-1871.

[9]. Canpolat L, Kukner A, Canpolat I, Ozan E (1999) Ultrtastructural and morphometric analysis of peripheral nerve regeneration within silicone tubes. Tr J of Medical Sciences 29(3): 203-209.

[10]. Chen YS, Hsieh CL, Tsai CC, Chen TH, Cheng WC, et al. (2000) Peripheral nerve regeneration using silicone rubber chambers filled with collagen, laminin and fibronectin.. Biomaterials 21(15): 1541-1547.

[11]. Lundborg G, Rosen B, Abrahamson SO, Dahlin L, Danielsen N (1994) Tubular repair of the median nerve in the human forearm, Preliminary findings. J hand Surg (Br) 19(3): 273-276.

[12]. Pfister LA, Papaloizos M, Merkle HP, Gander B (2007) Nerve conduits and growth factor delivery in peripheral nerve repair. J Peripher Nerv Syst 12(2): 65-82.

[13]. Feldman EL, Sullivan KA, Kim B, Russell JW (1999) Insulin-like growth factors regulate neuronal differentiation and survival. Neurobiol Dis 4(3): 201-214.

[14]. Kim B, Leventhal PS, Saltiel AR, Feldman EL (1997) Insulin-like growth factor-I-mediated neurite outgrowth in vitro requires MAP kinase activation. J Biolo Chem, 272(34): 1268-1273.

[15]. Matthews CC, Odeh H, Feldman EL (1997) Insulin-like growth factor-I is an osmoprotectant in human neuroblastoma cells. Neuroscience 79(2): 525-534.

[16]. Hansson HA, Nilsson A, Isgaard J, Billig H, Isaksson O, et al. (1988) Immunohistochemical localization of insulinlike growth factor I in the adult rat. Histochemistry 89(4): 403-410.

[17]. Hansson HA, Rozell B, Skottner A (1987) Rapid axoplasmic transport of insulin-like growth factor I in the sciatic nerve of adult rats. Cell Tissue Res 247(2): 241-247.

[18]. Bothwell M (1982) Insulin and somatomedin MSA promote nerve growth factor-independent neurite formation by cultured chick dorsal root ganglionic sensory neurons. J Neurosci Res 8(2-3): 225-231.

[19]. Bain JR, Mackinnon SE, Hunter DA (1989) Functional evaluation of complete sciatic, peroneal, and posterior tibial nerve lesions in the rat. Plast Reconstr Surg 83(1): 129-136.

[20]. Kimpinski K, Mearow K (2001) Neurite growth promotion by nerve growth factor and insulin-like growth factor-1 in cultured adult sensory neurons: role of phosphoinositide 3-kinase and mitogen activated protein kinase. J Neurosci Res 63(6): 486-499. 
[21]. Scolnick JA, Cui K, Duggan CD, Xuan S, Yuan XB, et al. (2008) Role of IGF signaling in olfactory sensory map formation and axon guidance. Neuron 57(6): 847-857.

[22]. Caroni P, Grandes P (1990) Nerve sprouting in innervated adult skeletal muscle induced by exposure to elevated levels of insulin-like growth factors. J Cell Biol 110(4): 1307-1317

[23]. Rosen CJ, Pollak M (1999) Circulating IGF-1: New perspectives for a new century. Trends Endo Metab 10(4): 136-141.

[24]. Solman SR, Baxter RC (1996) Insulin-like growth factor binding protein-3: factors affecting binary and ternary complex formation. Growth Reg 6(1): 42-47.

[25]. Twing SM, Baxter RC (1998) Insulin-like growth factor (IGF)-binding protein 5 forms an alternative ternary complex with IGFs and the acid-labile subunit. J Biological Chem 273(11): 6074-6079.

[26]. Anlar B, Sullivan KA, Feldman EL (1999) Insulin-like growth factor-I and central nervous system development. Hormone Metab Res 31(2-3): 120125.

[27]. Leventhal PS, Russell JW, Feldman EL, et al. (1999) IGFs and the nervous system. In: Contemporary endocrinology: the IGF system. Humana Press 17: 425-455.

[28]. Cheng HL, Feldman EL (1997) Insulin-like growth factor-I (IGF-I) and IGF binding protein-5 in Schwann cell differentiation. J Cell Physiol 171(2): 161-167.

[29]. Schumacher M, Jung-Testas I, Robel P, Baulieu EE (1993) Insulin-like growth factor I: a mitogen for rat Schwann cells in the presence of elevated levels of cyclic AMP. Glia 8(4): 232-240

[30]. Stewart HJS, Bradke F, Tabernero A, Morrell D, Jessen KR, et al. (1996) Regulation of rat Schwann cell Po expression and DNA synthesis by insulinlike growth factors in vitro. Eurp J Neurosci 8(3): 553-564.

[31]. Russell JW, Cheng HL, Golovoy D (2000) Insulin-like growth factor-I promotes myelination of peripheral sensory axons. J Peuropathol Exper Neurol 59(7): 575-584.

[32]. Zimmermann M (1983) Ethical guidelines for investigations of experimental pain in conscious animals. Pain 16(2): 109-110.

[33]. Basso DM, Beattie MS, Bresnahan JC (1995) A sensitive and reliable locomotor rating scale for open field testing in rats. J Neurotrauma 12(1): 1-21.

[34]. Bervar M (2000) Video analysis of standing-an alternative footprint analysis to assess functional loss following injury to the rat sciatic nerve. J Neurosci Methods 102(2): 109-116.

[35]. Geuna S, Gigo-Benato D, Rodrigues AC (2003) On sampling and sampling errors in histomorphometry of peripheral nerve fibers. Microsurg 24(1): 7276.

[36]. Terada M,Yasuda H, Kikkawa R (1998) Delayed Wallerian degeneration and increased neurofilament phosphorylation in sciatic nerves of rats with streptozocin-induced diabetes. J Neurol Sci 155(1): 23-30.

[37]. Terada M, Yasuda H, Kogawa S, Maeda K, Haneda M, et al. (1998) Expression and activity of cyclin-dependent kinase 5/p35 in adult rat peripheral nervous system. J Neurochem 71(6): 2600-2606.

[38]. Yasuda H, Terada M, Maeda K, Kogawa S, Sanada M, et al.(2003) Diabetic neuropathy and nerve regeneration. Prog Neurobiol 69: 229-285.

[39]. Dellon AL, Mackinnon SE (1989) Sciatic nerve regeneration in the rat. Validity of walking track assessment in the presence of chronic contractures. Microsurg 10(3): 220-225.

[40]. Shen N, Zhu J (1995) Application of sciatic functional index in nerve functional assessment. Microsurg 16(8): 552-555.

[41]. Kanaya F, Firrell JC, Breidenbach WC (1996) Sciatic function index, nerve conduction tests, muscle contraction, and axon morphometry as indicators of regeneration. Plast Reconstr Surg 98(7): 1264-1274.

[42]. Mackinnon SE, Hudson AR, Hunter DA (1985) Histologic assessment of the nerve regeneration in the rat. Plast Reconstr Surg 75: 384-388.

[43]. Almquist E, Eeg-Olofsson O (1970) Sensory-nerve-conduction velocity and two-point discrimination in sutured nerves. J Bone Joint Surg 52(4): 791 796.

[44]. De Medinaceli L, Freed WJ, Wyatt RJ (1982) An index of the functional condition of rat sciatic nerve based on measurements made from walking tracks. Exp Neurol 77(3): 634-643.

[45]. Varej־ao ASP, Melo-Pinto P, Meek MF, Filipe VM, Bulas-Cruz J (2004) Methods for the experimental functional assessment of rat sciatic nerve regeneration. Neurol Res 26(2): 186-194.

[46]. Castaneda F, Kinne RK (2002) Omental graft improves functional recovery of transected peripheral nerve. Muscle Nerve 26(4): 527-532.

[47]. Azizi S, Mohammadi R, Amini K, Fallah R, Karegar K (2010) Bridging small-gap peripheral nerve defect using silicone rubber chamber in the rat sciatic nerve transection model. Vet Res Forum 1(2): 107-115.

[48]. Nachemson AK, Lundborg G, Hansson HA (1990) Insulin-like growth factor I promotes nerve regeneration: an experimental study on rat sciatic nerve. Growth Factors 3(4): 309- 314.

[49]. Kanje M, Skottner A, Sjoberg J, Lundborg G (1989) Insulin-like growth factor I (IGF-I) stimulates regeneration of the rat sciatic nerve. Brain Res 486(2): 396-398.

[50]. Streppel M, Azzolin N, Dohm S, Guntinas-Lichius O, Haas C, et al. (2001) Focal application of neutralizing antibodies to soluble neurotrophic factors reduces collateral axonal branching after peripheral nerve lesion. Eur J Neurosci 15(8): 1327-1342.

[51]. Kanje M, Skottner A, Lundborg G, Sjöberg J (1991) Does insulin-like growth factor I (IGF-1) trigger the cell body reaction in the rat sciatic nerve? Brain Res 563(1-2): 285-287. 